\title{
Systematic Methods for Exploiting Temperature-Dependent Phenomena in Chemiresistive Sensing
}

\author{
P. H. Rogers, K. D. Benkstein, S. Semancik \\ National Institute of Standards and Technology, Biochemical Science Division, \\ 100 Bureau Drive, MS 8362, Gaithersburg, MD 20899-8362, USA \\ steves@nist.gov
}

\begin{abstract}
:
Addressable elements within chemiresistive microsensor arrays have been operated with rapid temperature modulation in order to improve their ability to detect and monitor species of interest. This approach involves purposeful manipulation of surface adsorbate populations that significantly enhances device selectivity, allowing increasingly complex analyses. In the present work involving three types of oxide sensing films, we focus on systematic methods that have been used to develop materials-specific temperature programs which are tuned for recognition and quantification of multiple trace compounds in test mixtures within air backgrounds.
\end{abstract}

Key words: breath analysis, chemical microsensor, oxides, sensor array, temperature

\section{Introduction}

Real world chemical analysis problems present significant challenges for electronic nose sensing technologies, and a variety of methods (multielement arrays, combinations of transduction principles, modulation techniques, etc.) have been used in efforts to achieve the required levels of measurement sensitivity and selectivity [1]. Temperature has been recognized as an important parameter that can affect transduction processes, and therefore signal information content. While temperature is often fixed in chemiresistor sensor operation, micromachined microhotplate sensing platforms have enabled temperature control with rapid $(<1 \mathrm{~s})$ thermal excursions. Work involving pseudo-random temperature variation was performed with microhotplates to identify particular temperature regimes where two similar molecules could be differentiated in an air-only background [2]. Temperature programming has also been used to enrich signal streams for recognizing individual chemical hazards in air which was sometimes spiked with interfering molecules [3], and hierarchical analysis methods have been employed with temperature-modulated data to successfully recognize and classify individual presentations (into an air background) of single analytes [4].

In this presentation, we focus on methods for the acquisition and analysis of chemiresistive signals that are tailored for recognition, as well as quantification, of multiple trace compounds, even when the targets are simultaneously presented in air-based mixtures. Our tuning approach utilizes systematic, base-shifted, temperature programs to acquire response databases. Data reduction methods and custom selection algorithms are applied to produce sensing-materials-specific and time-shortened thermal programs, which allow one to acquire the most useful analytical information with shorter collection times. The resulting capabilities rely on transient and historydependent interfacial phenomena, related to thermodynamic and kinetic effects of adsorption, desorption and interactions for adsorbates and coadsorbates. While we believe the optimized, thermal-control methodology has implications for a spectrum of sensing applications, the capabilities are demonstrated in this paper for a breath biomarker study on multiple target compounds.

\section{Experimental Approach}

A 16-element microhotplate array fabricated by top-side micromachining was used in this study; this type of platform has been described in detail elsewhere [2]. Sensing films of $\mathrm{SnO}_{2}$, $\mathrm{In}_{2} \mathrm{O}_{3}$ and $\mathrm{CuO}$ were formed on device elements by localized annealing of micropipetted metal-hydroxide sols. The metalhydroxide solutions were synthesized using two different methods. For tin hydroxide, ammonium hydroxide solution was slowly added to an aqueous solution of $\mathrm{SnCl}_{4}$ following procedures 


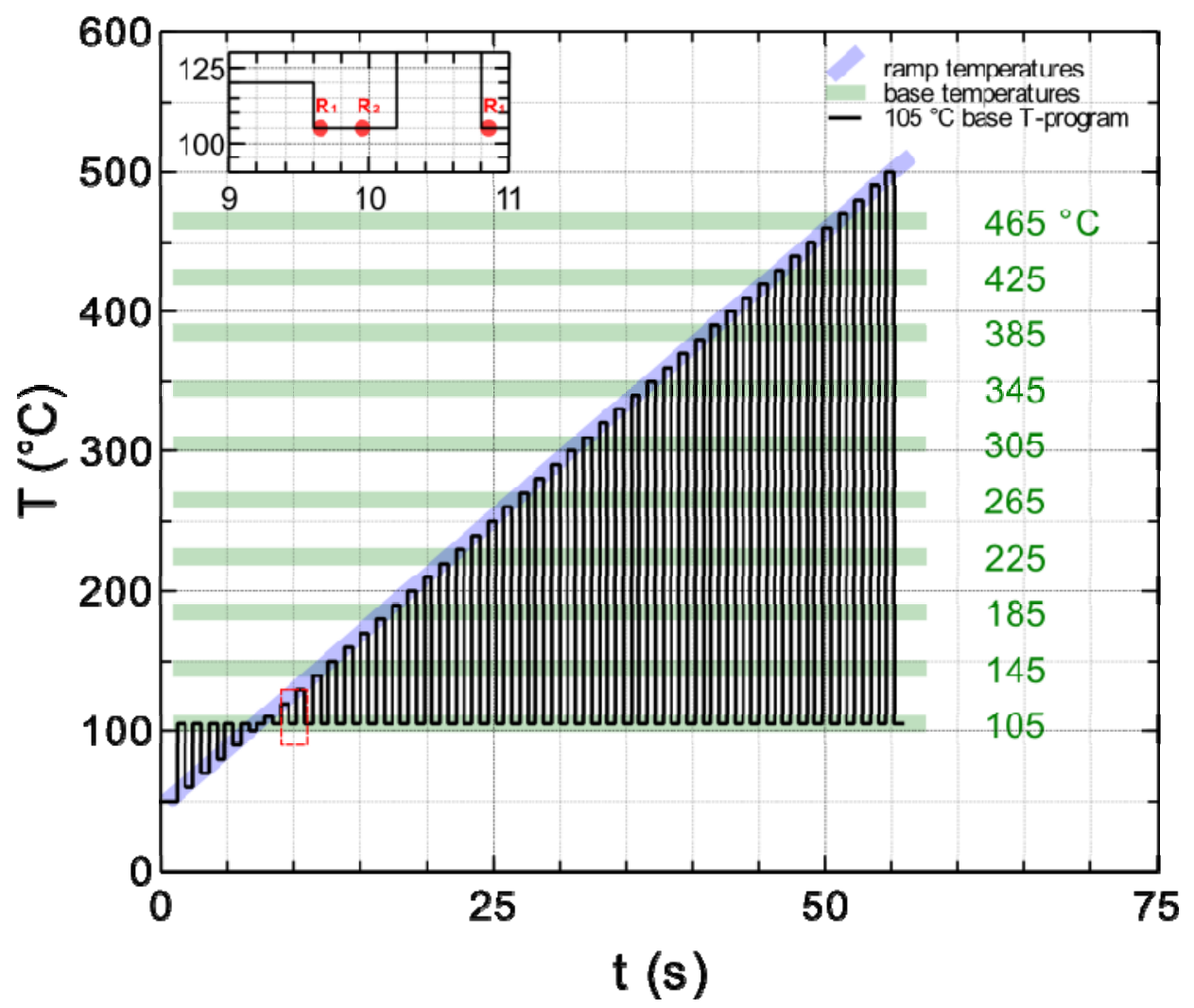

Fig. 1: Temperature cycling used to acquire optimization database, with different base temperatures shown in green (see also [6]). The $R_{1}$ and $R_{2}$ points in the inset show examples of when resistance measurements were made during the base temperature step.

reported previously [5]. Indium hydroxide and copper hydroxide solutions were synthesized separately by the electrolysis of water with $99.998+\%$ pure metals as the anodes and aluminum foil as the cathode. Solution droplets estimated to be $\approx 650 \mathrm{pL}$ were administered to individual suspended microhotplate elements using a $75 \mu \mathrm{m}$ inner-diameter polycarbonate microcapillary tube. Gentle heating of the microhotplates was performed to remove volatiles, and the deposition process was repeated two more times, to attain thicker oxide films, before annealing to $100{ }^{\circ} \mathrm{C}, 300^{\circ} \mathrm{C}$ and $500{ }^{\circ} \mathrm{C}[6]$.

The systematic temperature cycling used to characterize temperature-dependent responses of the three types of chemiresistive oxide microsensors to gas exposures is shown schematically in Figure 1 . The pulsed temperature ramp was altered over the course of data collection by offsetting the "base" settings sequentially in steps of $40^{\circ} \mathrm{C}$, as shown, from $105^{\circ} \mathrm{C}$ to $465^{\circ} \mathrm{C}$. For the purposes of this work, three target analytes were selected: acetone, ammonia and methanol (all disease biomarkers which can be found in breath), and they were injected at concentrations of $5 \mu \mathrm{mol} / \mathrm{mol}, 10 \mu \mathrm{mol} / \mathrm{mol}$ and $20 \mu \mathrm{mol} / \mathrm{mol}$ into air-based backgrounds. The microsensor array was exposed during studies in a total gas flow of $1000 \mathrm{~cm}^{3} / \mathrm{min}$.
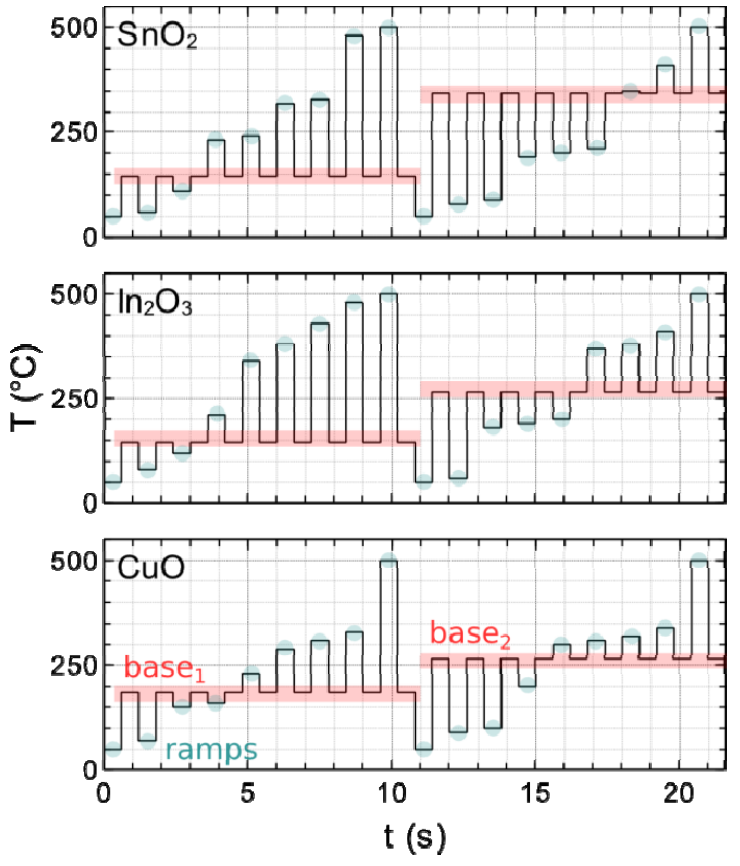

Fig. 2: Materials-tailored temperature programs for the three target analytes described in this work.

Chemiresistive data collection over multiple temperature cycles for each specific program produces repeating patterns of resistance 


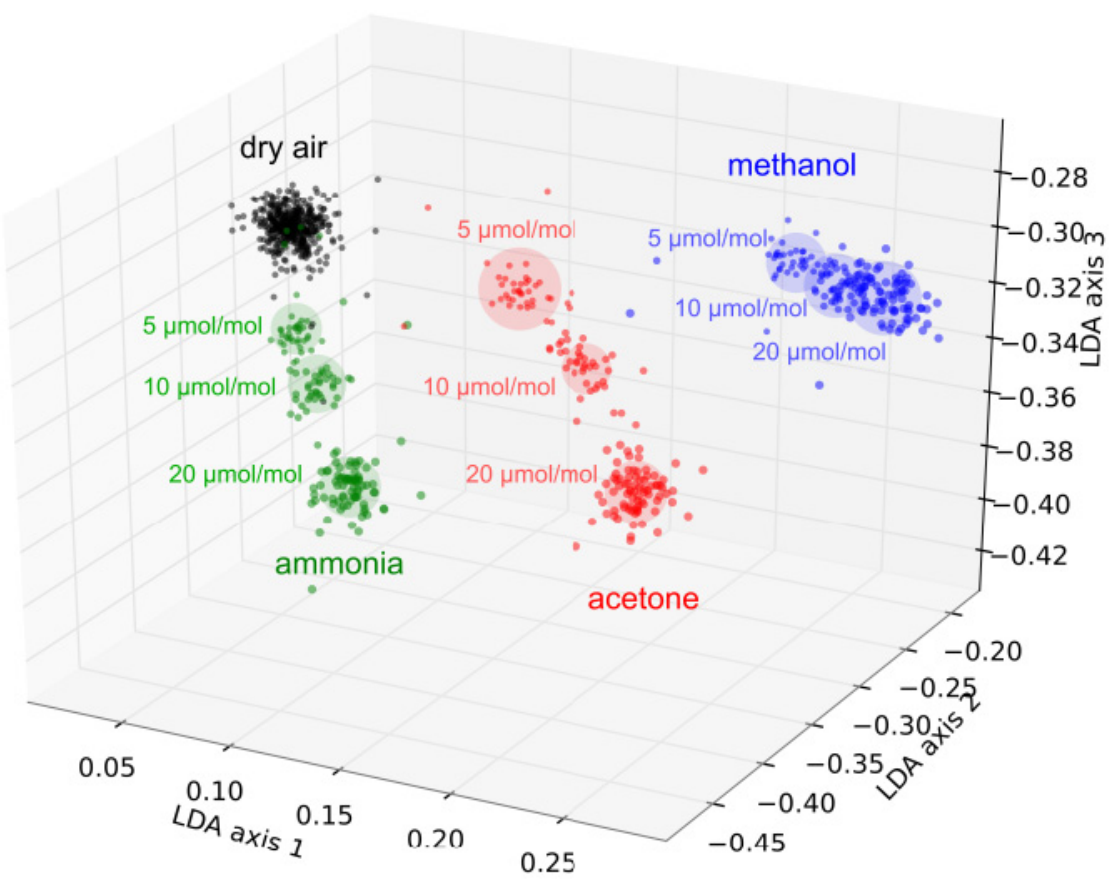

Fig. 3: LDA plot showing clustering of dry air and target species with varied concentrations.

measurements from each type of sensing element within the array. We used linear discriminant analysis (LDA), cluster separability and principle component analysis (PCA) biplots to extract information from the acquired databases, and identify specific temperature bases and excursions contributing the most to analyte and concentration discrimination [6].

\section{Results}

The resulting materials-specific temperature programs used for sensing acetone, ammonia and methanol are shown in Figure 2. These tailored programs (from design protocols constrained to include two base temperature values, with nine pulsed temperature excursions each) are relatively short in time and unique for each oxide. These programs offer recognition and quantification for acetone, ammonia and methanol derived from LDA processing (Figure 3 ) that is consistently superior to recognition/quantification possible when employing different programs (such as subsets from the cycles depicted in Figure 1). Note that each analyte and the air background cluster in separate portions of the LDA plot, and that there is good separation of the three target concentrations for acetone and ammonia.

The microdevice operated with these "optimized" programs was then employed in a separate study [7] where one, two or three of these disease-biomarker targets were presented in simulated breath backgrounds (consisting of $77 \% \mathrm{~N}_{2}, 3 \%$ to $7 \% \mathrm{CO}_{2}$, balanced by $\mathrm{O}_{2}$ ) with $65 \%$ relative humidity. The target analyte concentrations varied between $5 \mu \mathrm{mol} / \mathrm{mol}$ and $20 \mu \mathrm{mol} / \mathrm{mol}$ (levels in the general range related to detection/monitoring of homeostatic imbalances). While the simulated breath background differs somewhat from the air background used in optimization, it still contains $\approx 13 \%$ to $17 \% \quad \mathrm{O}_{2}$. This level is sufficient to maintain similar mechanistic behavior in the operation of oxide-based chemiresistive sensors in this study. The application of the temperature programs in Figure 2 was found to produce data that yield good separability for all presentations of the three biomarkers in simulated breath, as shown in Figure 4. In addition, analysis of the response data for the varied concentrations of the targets has allowed regression-based quantification of the biomarkers.

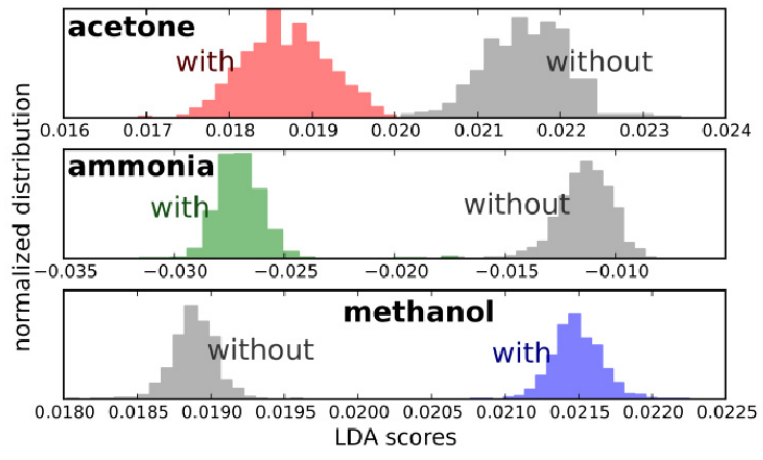

Fig. 4: Separability of target-containing exposures at any non-zero concentration from all other exposures for biomarkers in simulated breath. 


\section{Conclusion}

A key aspect of the approach described, and demonstrated here for breath biomarkers, is the use of tuned temperature modulation to obtain valuable analytical data with shorter acquisition times. By down-selecting to the most relevant temperature excursions for operation, the associated signal processing becomes not only more successful in recognition and quantification, but also easier. We believe this type of methodology can be utilized for a wide range of sensing and monitoring problems.

\section{Acknowledgements}

PHR was supported by a National Institute of Standards and Technology Postdoctoral Associateship Award administered through the National Research Council.

\section{References}

[1] F. Roeck, N. Barsan and U. Weimar, Electronic Nose; Current Status and Future Trends, Chemical Reviews 108, 705-725 (2008); doi:10.1021/cr068121q
[2] T. A. Kunt, T. J. McAvoy, R. E. Cavicchi and S. Semancik, Optimization of Temperature Programmed Sensing for Gas Identification Using Microhotplate Sensors, Sensors and Actuators B 53, 24-43 (1998); doi:10.1016/S0925-4005(98)00244-5

[3] B. Raman, D. C. Meier, J. K. Evju and S. Semancik, Designing and Optimizing Microsensor Arrays for Recognizing Chemical Hazards in Complex Environments, Sensors and Actuators B 137, 617-629 (2009); doi: 10.1016/j.snb.2008.11.053

[4] B. Raman, J. L. Hertz, K. D. Benkstein, and S. Semancik, Bioinspired Methodology for Artificial Olfaction, Anal. Chem. 80, 8364-8371 (2008); doi: 101021/ac8007048

[5] B. Ghaddab, F. Berger, J. B. Sanchez, P. Menini, C. Mavon, P. Yoboue, Benzene Monitoring by Micromachined Sensors with $\mathrm{SnO}_{2}$ Layer Obtained by Using Microdroplet Deposition Technique Sensors and Actuators B 152, 68-72 (2011); doi: 10.1016/j.snb.2010.09.046

[6] P. H. Rogers and S. Semancik, Development of Optimization Procedures for Application-Specific Chemical Sensing, Sensors and Actuators B 163, 8-19 (2012); doi:10.1016/j.snb.2011.11.015

[7] P. H. Rogers, K. D. Benkstein and S. Semancik, Sensing Multiple Biomarkers in Simulated Breath Using a Temperature-Pulsed e-nose, (in preparation) 